\title{
ION CHANNELING ANALYSIS OF GALLIUM NITRIDE IMPLANTED WITH
} DEUTERIUM

\author{
W. R. Wampler and S. M. Myers \\ Sandia National Laboratories, Albuquerque, NM 87185-1056, wrwampl@sandia.gov
}

\section{Cite this article as MRS Internet J. Nitride Semicond. Res., 4S1, G3.73 (1999)}

\begin{abstract}
Ion channeling and transmission electron microscopy were used to examine the microstructure of GaN implanted with deuterium (D) at high ( $>1$ at. \%) and low $(<0.1$ at. \%) D concentrations. At high concentrations, bubbles and basal-plane stacking faults were observed. Ion channeling showed the $\mathrm{D}$ was disordered relative to the $\mathrm{GaN}$ lattice, consistent with precipitation of $\mathrm{D}_{2}$ into bubbles. At low $\mathrm{D}$ concentrations, bubbles and stacking faults are absent and ion channeling shows that a large fraction of the D occupies sites near the center of the c-axis channel.
\end{abstract}

\section{INTRODUCTION}

Hydrogen is incorporated into GaN during growth by MOCVD and also during subsequent processing [1]. This hydrogen strongly affects the electrical properties by passivation of dopants and lattice defects. Release of hydrogen from $\mathrm{GaN}$ films requires annealing at temperatures above $800^{\circ} \mathrm{C}$ [2]. However, large reductions in resistivity of $\mathrm{Mg}$ doped $\mathrm{GaN}$ can be achieved by annealing at lower temperatures where $\mathrm{H}$ is not released from the material [3,4]. This indicates that the atomic configuration of $\mathrm{H}$ within the $\mathrm{GaN}$ lattice significantly affects its influence on electrical properties.

Here we use ion channeling to examine the lattice location of deuterium implanted into wurtzite GaN. Results from the channeling measurements are compared with first-principles calculations of the lattice configuration of hydrogen in GaN $[5,6]$. In addition, we use ion channeling and TEM to examine lattice defects produced by the implantation of deuterium.

\section{EXPERIMENTAL PROCEDURES}

Wurtzite GaN films with (0001) orientation and thickness in the range of $1.4-2.3 \mu \mathrm{m}$ were grown epitaxially by MOCVD on $420 \mu \mathrm{m}$ thick c-oriented sapphire substrates as described elsewhere [7]. The GaN was n-type with a carrier density of $\sim 10^{17} / \mathrm{cm}^{3}$ as determined by conductivity and Hall-effect measurements. The samples were implanted at room temperature with deuterium (D) at $50 \mathrm{keV}$ to fluences of $10^{15} / \mathrm{cm}^{2}$ and $10^{17} / \mathrm{cm}^{2}$. This gives D concentration profiles peaking $0.4 \mu \mathrm{m}$ beneath the surface at concentrations of about 0.05 and 5 atomic $\%$ for these two fluences [8].

Ion channeling measurements were done with the samples mounted on a 3 axis goniometer. D was analyzed by counting protons from the $\mathrm{D}\left({ }^{3} \mathrm{He}, \mathrm{p}\right) \alpha$ nuclear reaction with an incident beam of $0.85 \mathrm{MeV}^{3} \mathrm{He}^{+}$ions. With this energy $\mathrm{D}$ at depths up to about $1 \mu \mathrm{m}$ will be detected and the peak in the nuclear reaction cross section [8] at $0.6 \mathrm{MeV}$ occurs at the depth of the D. Analysis beams of $2 \mathrm{MeV}^{4} \mathrm{He}$ were used to examine lattice damage caused by the D implantation. Energy spectra of ${ }^{4} \mathrm{He}$ backscattered at 155 degrees were recorded for angles of incidence near the c-axis. The analysis beam size was typically $1 \mathrm{~mm}$ square and the angular divergence was 0.05 degree.

\section{EXPERIMENTAL RESULTS AND INTERPRETATION Lattice defects due to $D$ implantation}


Transmission electron microscopy, both plan view and cross section, shows that GaN implanted with $50 \mathrm{keV} \mathrm{H}$ to fluences of $2 \times 10^{16} / \mathrm{cm}^{2}$ and $10^{17} / \mathrm{cm}^{2}$ and then vacuum annealed at $886^{\circ} \mathrm{C}$ for one hour, contains cavities with a typical size of $10 \mathrm{~nm}$ at the $\mathrm{H}$ implant depth [8]. These cavities are believed to result from precipitation of $\mathrm{H}_{2}$ gas. Such samples also contain planar defects typically $\sim 50 \mathrm{~nm}$ in diameter, which are shown by high resolution imaging [8] to be (0001) basal plane stacking faults containing one extra $\mathrm{Ga}-\mathrm{N}$ bilayer bounded by a partial edge dislocation loop with a Burgers vector c/2[0001]. The atomic configuration of such faults has been discussed elsewhere [9]. TEM showed these cavities and stacking faults were not present in $\mathrm{GaN}$ implanted with $\mathrm{H}$ to a lower fluence of $10^{15} / \mathrm{cm}^{2}$ and annealed at $886^{\circ} \mathrm{C}$. The cavities and stacking faults can be seen in the micrographs shown in figure 1.
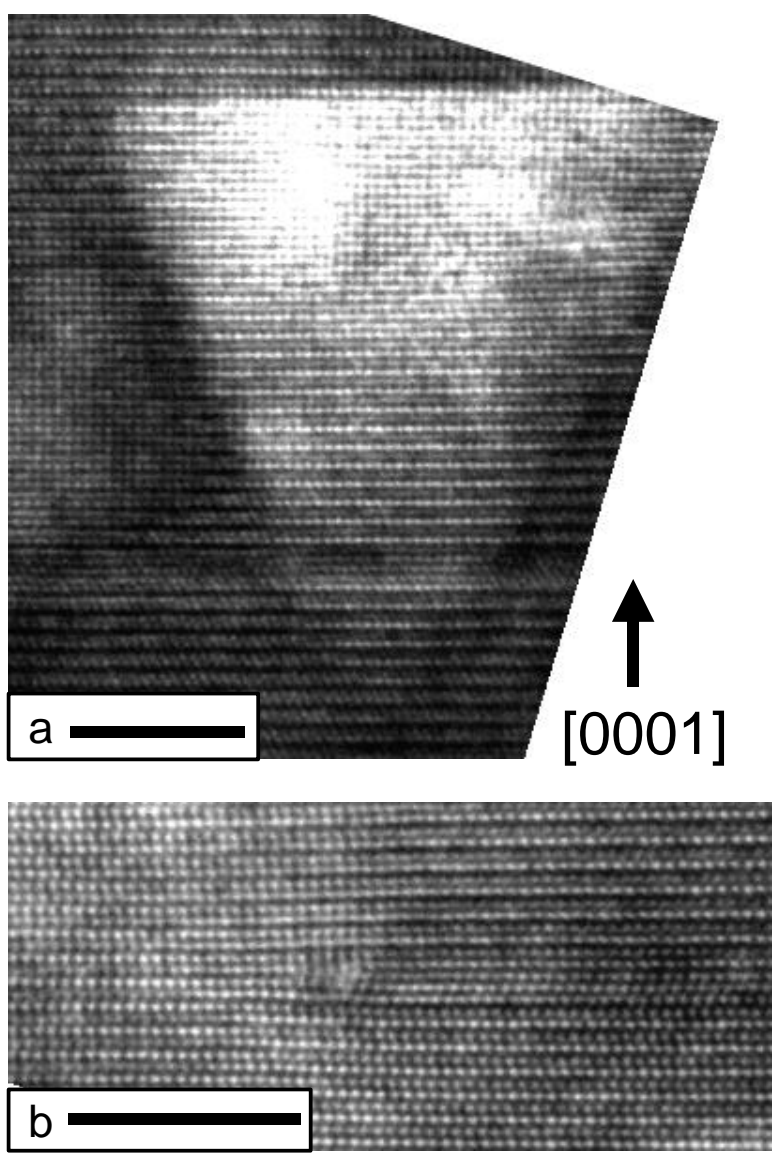

Fig. 1. Cross-section TEM of a bubble (a) and a stacking fault (b) in $\mathrm{GaN}$ implanted with $2 \times 10^{16} \mathrm{H} / \mathrm{cm}^{2}$ and then vacuum annealed at $886^{\circ} \mathrm{C}$ for one hour. The bars indicate $5 \mathrm{~nm}$.

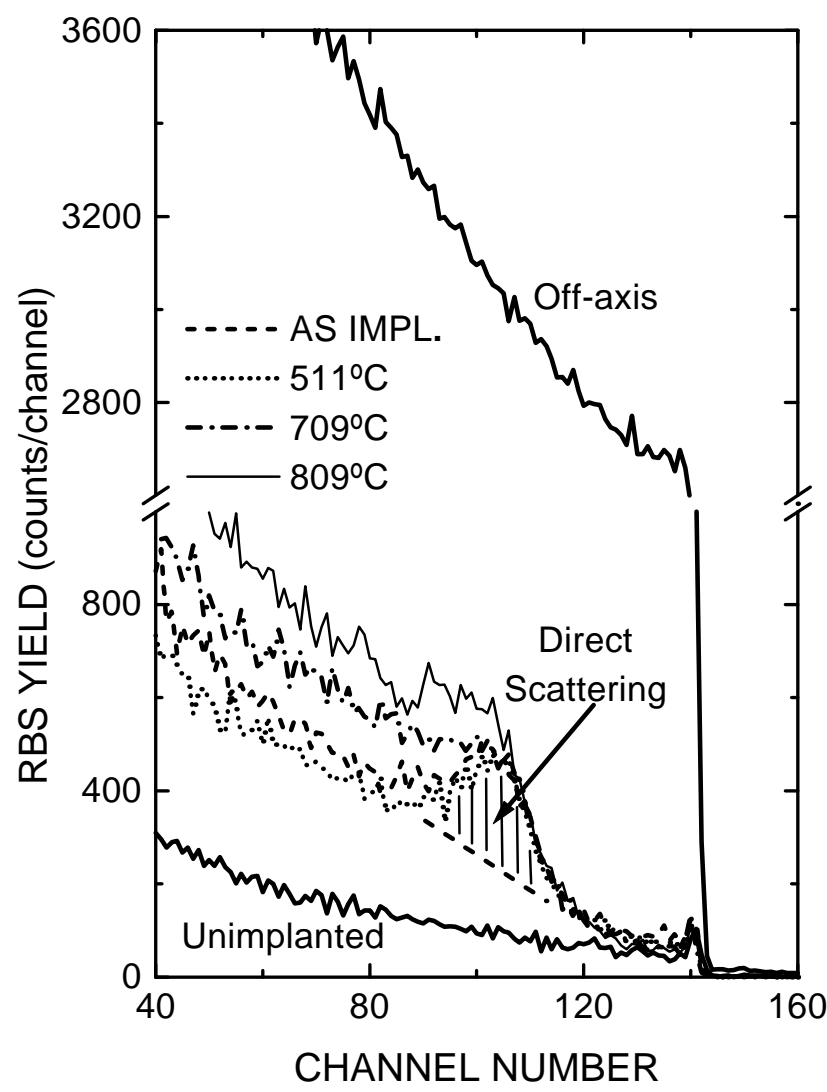

Fig. 2. Energy spectra for channeled $2 \mathrm{MeV}$ ${ }^{4} \mathrm{He}$ backscattered from $\mathrm{GaN}$. Yield from the implanted region appears at channels in the range from 90-120, while the surface corresponds approximately to channel 140 .

Figure 2 shows channeled backscattering spectra for $2 \mathrm{MeV}{ }^{4} \mathrm{He}$ incident along the [0001] axis for GaN implanted at room temperature with $10^{17} \mathrm{D} / \mathrm{cm}^{2}$. Also shown for comparison is a spectrum for an off-axis unchanneled or random direction obtained by averaging spectra taken at several off-axis orientations, and a channeled spectrum taken on a sample as-grown, i.e. not implanted with D. The channeled spectrum for a low-dose $\left(10^{15} / \mathrm{cm}^{2}\right)$ implanted sample (not shown) was the same as those for unimplanted samples. The low backscattering yield for axial channeling in the unimplanted $\mathrm{GaN}$ of $\sim 2 \%$ of random yield near the surface and $3.2 \%$ of random yield at the depth of $\mathrm{D}$ implantation, is consistent with a film which has few lattice defects. 
Room-temperature implantation of $10^{17} \mathrm{D} / \mathrm{cm}^{2}$ at $50 \mathrm{keV}$ creates a direct scattering peak, which is shaded in the figure, at an energy corresponding to He scattered from $\mathrm{Ga}$ at the depth of the implanted D, and increases the yield at lower energies due to dechanneling. Subsequent one hour vacuum anneals in the temperature range $511-709^{\circ} \mathrm{C}$ removes the direct scattering peak and increases the dechanneling step. The direct scattering peak shows that prior to annealing, Ga atoms are displaced from lattice sites into the open [0001] channel, thereby enhancing the backscattering yield of channeled ${ }^{4} \mathrm{He}$ ions. We refer to these displaced $\mathrm{Ga}$ atoms generically as $\mathrm{Ga}$ interstitials without implying a specific lattice location. The disappearance of this direct scattering peak with annealing indicates a return to lattice sites. The associated increase in dechanneling is consistent with the formation of extended defects with large strain fields, eg. dislocations, which dechannel the ${ }^{4} \mathrm{He}$ with little direct scattering. Reference 10 gives more detailed discussion of defect analysis by ion channeling.

The area under the direct scattering peak can be used to calculate the areal density of Ga interstitials. The number of counts in the peak is given by

$$
\mathrm{Y}=\mathrm{N}_{\mathrm{He}} \sigma_{\mathrm{R}} \Omega \mathrm{N}_{\mathrm{i}} \mathrm{f}
$$

where $\mathrm{N}_{\mathrm{He}}$ is the number of incident ${ }^{4} \mathrm{He}$ ions, $\sigma_{\mathrm{R}}$ is the Rutherford differential cross section for scattering of ${ }^{4} \mathrm{He}$ from Ga, $\Omega$ is the solid angle subtended by the detector, $\mathrm{N}_{\mathrm{i}}$ is the areal density of interstitial $\mathrm{Ga}$ and $\mathrm{f}$ is the flux enhancement due to channeling at the location of the interstitial Ga. With $\mathrm{Y}=3500$ from the channeled spectrum before annealing (fig. 2) and $\mathrm{f}=2$ from computer simulations (discussed later) of ${ }^{4} \mathrm{He}$ channeling in $\mathrm{GaN}$ assuming all $\mathrm{Ga}$ interstitials are near the center of the [0001] channel, we obtain $\mathrm{N}_{\mathrm{i}}=3.0 \times 10^{16} / \mathrm{cm}^{2}$. This number is smaller by two orders of magnitude than the areal density of collisional displacements caused by the D implantation, which is estimated to be $\sim 40$ displacements per incident ion or $4 \times 10^{18}$ displacements $/ \mathrm{cm}^{2}$. However, the areal density of $\mathrm{Ga}$ interstitials estimated from the direct scattering peak is close to the areal density $3.5 \times 10^{16} / \mathrm{cm}^{2}$ of $\mathrm{Ga}$ atoms displaced by the bubbles [8]. This result suggests the following model for bubble formation: the vacancies and interstitials produced by atomic displacements mostly recombine, however a small fraction of the vacancies agglomerate and combine with $\mathrm{H}$ to form high pressure $\mathrm{H}_{2}$ bubbles, leaving a corresponding number of interstitials in the neighboring lattice. At temperatures in the range from 500 to $700^{\circ} \mathrm{C}$ most of these interstitials annihilate, possibly by diffusing to the surface while some agglomerate into the stacking faults seen by TEM.

If it is assumed that the dechanneling step is due to strain fields from the dislocation loops bounding the stacking faults, then the number of these defects and the areal density of interstitial atoms enveloped by them can be estimated from the height of the dechanneling step. In general the areal density of a defect is related to dechanneling by the equation [10]

$$
\mathrm{N}_{\mathrm{D}}=\sigma_{\mathrm{d}}^{-1} \ln \left[\left(1-\chi_{\mathrm{v}}\right) /\left(1-\chi_{\mathrm{D}}\right)\right] \text {, }
$$

were $\chi_{\mathrm{D}}$ is the channeled backscattering yield above the step divided by the random or unchanneled yield, and $\chi_{\mathrm{v}}$ is the corresponding quantity in the absence of defects. The cross section for dechanneling by the strain field of a dislocation is given by [10]

$$
\sigma_{\mathrm{d}}=\mathrm{K}\left(\mathrm{a}_{\mathrm{TF}} \mathrm{b}\right)^{1 / 2} / \psi_{1}
$$

where $\mathrm{a}_{\mathrm{TF}}$ is the Thomas-Fermi screening distance, $\mathrm{b}$ is the length of the Burgers vector, $\psi_{1}$ is a critical angle for dechanneling. Using a value $\mathrm{K}=1.2$, derived elsewhere for axial channeling 
perpendicular to an edge dislocation [11], we obtain $\sigma_{\mathrm{d}}=5.3 \times 10^{-7} \mathrm{~cm}^{2} / \mathrm{cm}$. With $\chi_{\mathrm{v}}=0.032$ and $\chi_{\mathrm{D}}=0.19$ from the channeled spectrum after annealing at $809^{\circ} \mathrm{C}$, eq. 2 yields $\mathrm{N}_{\mathrm{D}}=3.4 \times 10^{-5}$ $\mathrm{cm} / \mathrm{cm}^{2}$. Assuming the stacking faults are circular with a diameter of $50 \mathrm{~nm}$ estimated from TEM, this value of $\mathrm{N}_{\mathrm{D}}$ implies that the associated areal density of extra Ga and $\mathrm{N}$ atoms is $1.0 \times 10^{15} / \mathrm{cm}^{2}$. This number is smaller by a factor of 70 than the areal density of Ga and $\mathrm{N}$ atoms calculated to be displaced by bubble formation.

We also conclude that the bubbles themselves do not contribute significantly to direct scattering and dechanneling. The combined basal plane area of the bubbles per unit sample area is estimated to be $\sim 3 \mathrm{~cm}^{2} / \mathrm{cm}^{2}$ [8]. If the He ions re-enter the lattice at random locations after traversing a bubble, the direct scattering and dechanneling from the bubbles should be about three times that of the external surface, which is small compared to the observed direct scattering and dechanneling as can be seen in figure 2 .

\section{Location of D in the GaN lattice}

The location of foreign atoms relative to the host lattice can be determined by ion channeling [10]. Here we use the $\mathrm{D}\left({ }^{3} \mathrm{He}, \mathrm{p}\right) \alpha$ nuclear reaction to study the lattice location of $\mathrm{D}$ implanted into $\mathrm{GaN}$. The yield is proportional to the local flux of ${ }^{3} \mathrm{He}$ at the location of the D. For angles of incidence far from major axes or planes the flux of ${ }^{3} \mathrm{He}$ is nearly the same at all locations in the lattice. However, when the analysis beam is aligned along the c-axis, channeling reduces the flux near the rows of host atoms and increases the flux near the center of the open channels relative to fluxes with off-axis alignment. This will give rise to a dip in the NRA yield if the D is near the host atom rows, for example at a substitutional site, or conversely, to a peak in the NRA yield if the $\mathrm{D}$ is near the center of the channel. The absence of a peak or dip would indicate that the $\mathrm{D}$ is randomly located relative to the lattice.

Figure 3 shows the measured NRA yield normalized to the off-axis or random yield versus the angle between the analysis beam direction and the caxis. For GaN implanted to the low dose of $10^{15} \mathrm{D} / \mathrm{cm}^{2}$ there is a narrow peak $\sim 40 \%$ above the random yield, whereas for $\mathrm{GaN}$ implanted with $10^{17}$ $\mathrm{D} / \mathrm{cm}^{2}$ there is no peak. The absence of a peak for the high dose implanted sample is consistent with the idea that at high concentrations most of the D precipitates as gas into cavities which would give random location for the $\mathrm{D}$

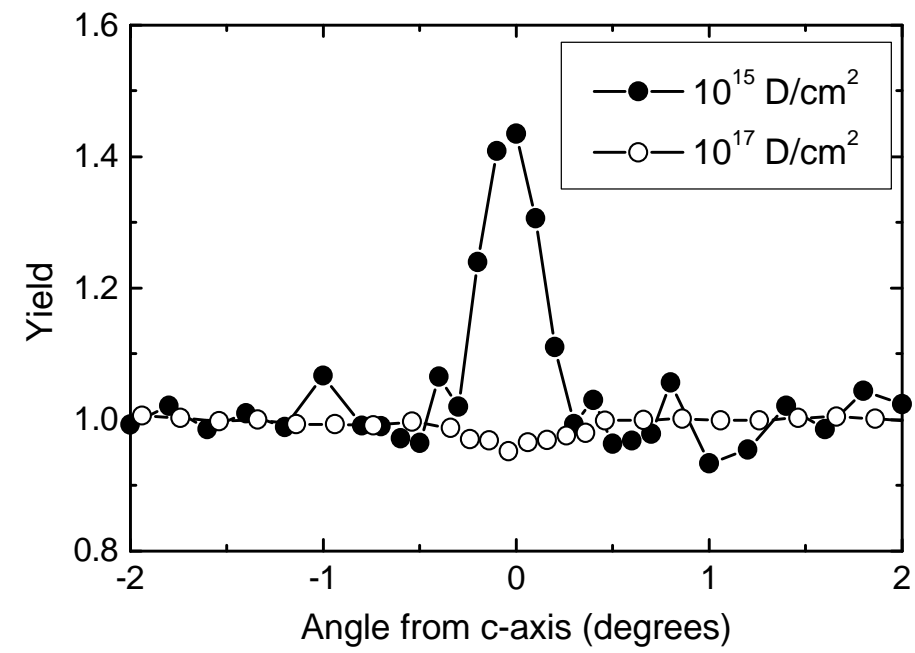

Fig. 3. NRA yield versus angle from the $c$ axis. relative to the GaN lattice sites. The peak for the low dose sample shows that a large fraction of the D is in the open channels. The height of this peak was observed to be a function of the analysis beam dose. Figure 4 shows the peak height as a function of off-axis ${ }^{3} \mathrm{He}$ analysis beam dose to the sample. The increase in peak height with beam dose shows that the analysis beam is causing the $\mathrm{D}$ to change its location in the lattice. The increase saturates after a few microcoulombs. The data shown in figure 3 were all taken after this beam induced increase had saturated. Measurements of the NRA yield after one hour vacuum anneals showed the peak remained unchanged up to $411^{\circ} \mathrm{C}$, decreased with increasing temperature above $511^{\circ} \mathrm{C}$, and became poorly resolved by $809^{\circ} \mathrm{C}$. 
We have carried out computer simulations of the yield versus angle for various D locations in the GaN lattice. These simulations were done using a statistical equilibrium continuum (SEC) model [12] modified for the case of channeling along the c-axis in wurtzite $\mathrm{GaN}$. The SEC model has previously been used to determine the lattice location of D implanted into silicon [12]. Our calculations use Doyle Turner potentials [13] for the GaN lattice with 24 rows. The model includes dechanneling due to thermal vibration of the host atoms. RMS vibrational amplitudes of $0.00735 \mathrm{~nm}$ for $\mathrm{Ga}$ and $0.00806 \mathrm{~nm}$ for $\mathrm{N}$ were used [14]. The

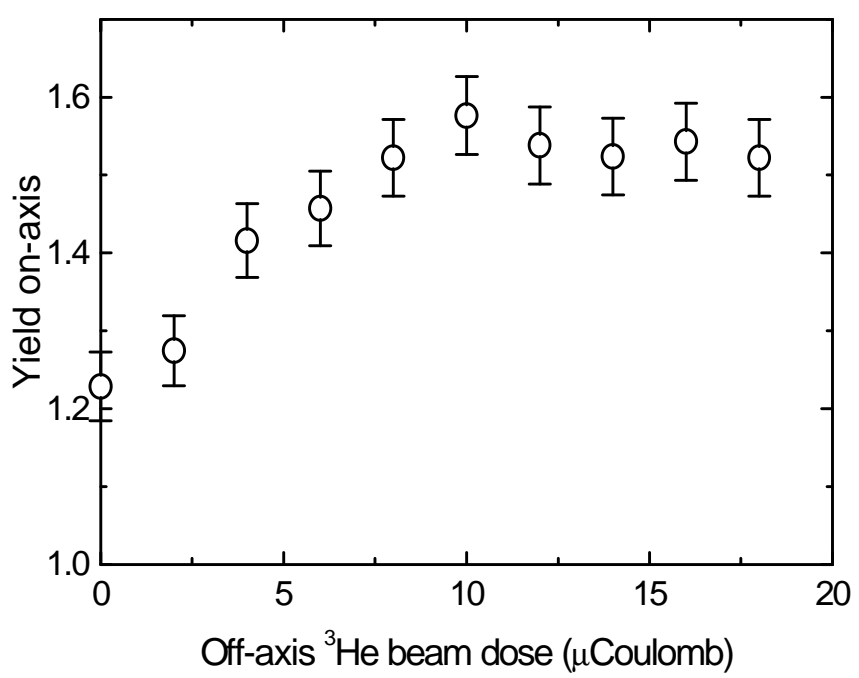

Fig. 4. NRA yield at axial alignment verses off-axis beam dose for GaN implanted with $10^{15} \mathrm{D} / \mathrm{cm}^{2}$.

SEC model gave good agreement with the observed channeling dip for $2 \mathrm{MeV}^{4} \mathrm{He}$ backscattered from the host lattice as shown in figure 5, providing an important validation of the model. The NRA yield curves were calculated using a vibrational amplitude of $0.010 \mathrm{~nm}$ for the $\mathrm{D}$. The $\mathrm{D}$ vibrational amplitude was estimated assuming a harmonic oscillator model with a vibrational frequency of $\sim 2300 \mathrm{~cm}^{-1}$ for the D-N stretch mode determined from infrared absorption measurements [15] and from first principles calculations [9]. Except for the S site, changes in D vibrational amplitude by factors of 2 do not significantly change the calculated yield curves. The yields calculated by the SEC model correspond to values averaged over all azimuthal angles. In order to compare with the SEC model, the data shown in figures 3 (NRA) and 4 (RBS) are averages of measurements at many azimuthal angles.

Figure 5 shows the yield predicted by the SEC model for various locations of $\mathrm{D}$ in the channel as indicated in the inset diagram. Curve $\mathrm{C}$ is for $\mathrm{D}$ at the channel center, curve $S$ is for $D$ in line with the host atom rows which includes substitutional sites. Also shown is the yield for $\mathrm{D}$ at a bond-center site midway between the $\mathrm{Ga}$ and $\mathrm{N}$ atoms at the channel edge. Another site which has been proposed for hydrogen in $\mathrm{GaN}$ is the nitrogen antibonding site which is along the tetrahedral bond direction $\sim 0.10 \mathrm{~nm}$ from the $\mathrm{N}$ atoms but opposite the neighboring $\mathrm{Ga}$ atoms [6]. The channeling yield curves for the one quarter of nitrogen antibonding and bond

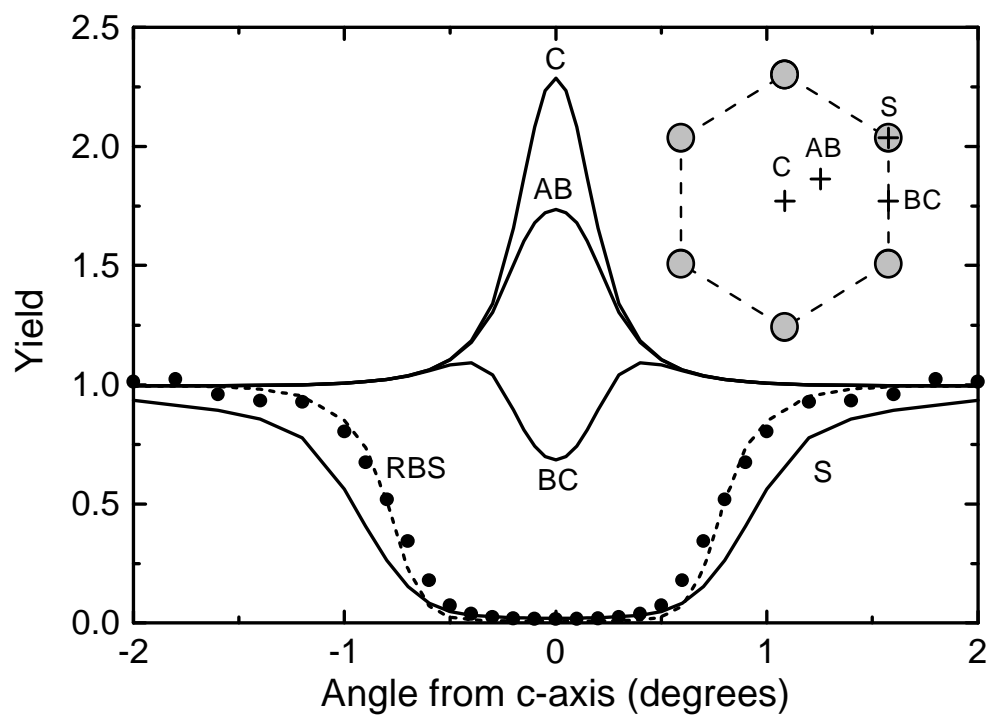

Fig. 5. Solid curves show the NRA yield versus angle calculated by the SEC model for D at the various indicated locations in the c-axis channel. The calculated backscattered yield (dashed curve) agrees well with measured azimuthally averaged yield (dots) of backscattered $2 \mathrm{MeV}^{4} \mathrm{He}$ 
center sites in-line with the host atom rows, are the same as for the $\mathrm{S}$ site. The yield curves for the remaining three quarters of these sites, at locations indicated in the inset diagram as BC and $\mathrm{AB}$ in the inset diagram, are shown in figure 5. In the wurtzite structure the two types of $\mathrm{AB}$ and $\mathrm{BC}$ sites are not equivalent and need not be equally occupied by $\mathrm{D}$. The peak we observe in the measured NRA yield shows that a large fraction, of order $50 \%$ or more, of the D occupies sites near the channel center such as the $\mathrm{C}$ or $\mathrm{AB}$ sites in the sample implanted with $10^{15} \mathrm{D} / \mathrm{cm}^{2}$.

The increase in NRA channeling yield with analysis beam dose which we observe, and the fact the D depth distribution does not broaden during thermal release, suggest that the $\mathrm{D}$ is bound at lattice defects. First-principles calculations have been reported for $\mathrm{H}$ bound to vacancies in $\mathrm{GaN}$ [6]. These calculations predict that $\mathrm{H}$ in a $\mathrm{N}$ vacancy will be located near the center of the vacancy. Accordingly, $\mathrm{D}$ trapped at $\mathrm{N}$ vacancies is expected to be located substitutionally at the $\mathrm{N}$ lattice site which would correspond to the $\mathrm{S}$ site shown in figure 5. Our NRA channeling data excludes significant occupation of $\mathrm{D}$ at this site.

$\mathrm{D}$ in a Ga vacancy should form a strong covalent bond with one of the $\mathrm{N}$ neighbors [6]. This would place the $\mathrm{D}$ about $0.10 \mathrm{~nm}$ from an $\mathrm{N}$ atom along the tetrahedral bond direction. Since the $\mathrm{GaN}$ bond length is $0.20 \mathrm{~nm}$ the predicted position for $\mathrm{D}$ in a Ga vacancy is therefore very close to the bond center location, in the absence of relaxation of the lattice around the vacancy. Therefore, the yield curves for $\mathrm{D}$ in a Ga vacancy for the $\mathrm{N}$ whose bond is along the c direction, should be similar to the curve for the S site. For D bound to the other three N, the yield curve should be similar to that of the $\mathrm{BC}$ site shown in figure 5. Both of these sites for $\mathrm{D}$ at a $\mathrm{Ga}$ vacancy give a dip in the channeling yield as shown in figure 5. In a recent study, IR absorption bands were observed due to $\mathrm{H}-\mathrm{N}$ and $\mathrm{D}-\mathrm{N}$ stretch mode vibrations in $\mathrm{GaN}$ implanted with $\mathrm{H}$ and $\mathrm{D}$ [15]. These absorption bands were tentatively assigned to $\mathrm{H}$ or $\mathrm{D}$ bound to $\mathrm{N}$ at $\mathrm{Ga}$ vacancies. Our channeling results show that most of the implanted D is not at such sites since this would give a dip in the NRA channeling yield in contrast to the peak we observe. It therefore seems appropriate to consider other defect-related sites, including interstitials or solution sites adjacent to defects as possible binding sites for D.

\section{ACKNOWLEDGMENTS}

The authors thank Jung Han for supplying the GaN and Alan Wright for insightful discussions of energy and structure calculations for hydrogen in GaN. This work was supported by the U.S. Department of Energy under contract DE-AC04-94AL8500, in part under auspices of the Office of Basic Energy Sciences. Sandia National Laboratories is a multi-program laboratory operated by Sandia Corporation, a Lockheed Martin Company, for the U.S. Department of Energy.

\section{REFERENCES}

1. S. J. Pearton, in GaN and related materials, edited by S. J. Pearton (Gordon and Breach, London, 1997) chapter 14.

2. J. M. Zavada, R. G. Wilson, C. R. Abernathy and S. J. Pearton, Appl. Phys Lett. 64, 2724 (1994).

3. M. Miyachi, T. Tanaka, Y. Kimura, H. Ota, Appl. Phys. Lett. 72, 1101 (1998).

4. S. J. Pearton, J. W. Lee, and C. Yuan, Appl. Phys. Lett. 68, 2690 (1996).

5. J. Neugebauer, and C. G. Van de Walle, Phys. Rev. Lett. 75, 4452 (1995).

6. C. G. Van de Walle, Phys. Rev. B56, 10020 (1997).

7. J. Han, T. B. Ng, R. M. Biefeld, M. H. Crawford, and D. M. Follstaedt. Appl. Phys Lett. 71, 3114 (1997). 
8. S. M. Myers, T. J. Headley, C. R. Hills, J. Han, G. A. Petersen, C. H. Seager and W. R. Wampler, MRS Internet J. Nitride Semicond. Res. 4S1,G5.8 (1999).

9. Z. Liliental-Weber, C. Kisielowski, S. Ruvimov, Y. Chen, J. Washburn, I Grzegory, M. Bockowski, J. Jun and S. Porowski, J. Electron. Mater. 25, 1545 (1996).

10. L. C. Feldman, J. W. Mayer and S. T. Picraux, Materials Analysis by Ion Channeling, (Academic, New York, 1982), pp. 88-135.

11. Y. Quéré, Phys. Stat. Sol. 30, 713 (1968).

12. B. Bech-Nielsen, Phys. Rev. B37, 6353 (1988).

13. P. A. Doyle and P. S. Turner, Acta Crystalogr. Sect.A 24, 390 (1968).

14. A. Yoshiasa, K. Koto, H. Maeda and T. Ishii, Jpn. J. Appl. Phys. 36, 781 (1997).

15. M. G. Weinstein, C. Y. Song, M. Stavola, S. J. Pearton, R. G. Wilson, R. J. Shul, K. P. Killeen and M. J. Ludowise, Appl. Phys Lett. 72, 1703 (1998). 